\title{
Analyse d'article
}

\author{
Olivier RAMPIN
}

Laboratoire de Neurobiologie des Fonctions Végétatives - UR 1060

INRA, Bât. 325, Jouy-en-Josas

Shingo T, Gregg C, Enwere E, Fujikawa H, Hassam R, Geary C, Cross JC, Weiss S. : Pregnancy-stimulated neurogenesis in the adult female forebrain mediated by prolactin. Science, 2003, 299 : 117-120.

Le cerveau adulte des mammifères est capable de fabriquer de nouvelles cellules nerveuses (neurogenèse) durant toute la vie. Ainsi dans le système olfactif de l'adulte, une population de cellules progénitrices (précurseurs des neurones) présente à la base du 3 ème ventricule se divise en permanence. Les cellules nouvellement formées migrent pour gagner le bulbe olfactif et se différencier en interneurones olfactifs. L'interruption de la migration des cellules engendre des déficits de la discrimination olfactive. La sensibilité olfactive est d'autant plus grande que l'on possède plus d'interneurones olfactifs dans le bulbe. L'olfaction est un sens chimique particulièrement important dans la reconnaissance et l'identification d'un partenaire sexuel, dans l'accouplement, ainsi que dans la reconnaissance des petits au sein d'une portée et les soins qui leurs sont apportés. On peut donc penser que le comportement sexuel agit d'une façon ou d'une autre sur la dynamique du renouvellement des interneurones olfactifs.

Dans le présent travail, les auteurs utilisent un marqueur cellulaire des interneurones nouvellement formés et suivent leur migration dans le système nerveux olfactif de souris femelles adultes durant la gestation. Au 7ème jour de gestation, le nombre des cellules progénitrices est supérieur de $65 \%$ à celui mesuré chez des souris non gestantes, dans cette seule région de la base du 3 ème ventricule. Une seconde augmentation de la production des cel-

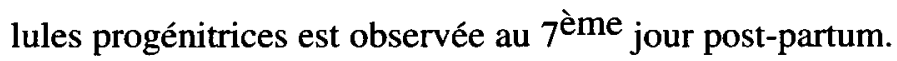
L'accouplement de souris avec des mâles vasectomisés accroît également la production des interneurones olfactifs, montrant que ce n'est pas l'implantation de l'embryon qui est à l'origine de ce mécanisme. L'injection d'estradiol ou de progestérone directement dans le cerveau de souris normales ou ovariectomisées n'a pas d'effet sur cette multiplication cellulaire. En revanche des injections périphériques ou centrales de prolactine miment les effets de la gestation sur les interneurones olfactifs.

Des expériences complémentaires réalisées in vitro permettent aux auteurs de montrer un effet de la prolactine sur la prolifération et la différenciation de neurones. Enfin chez des souris hétérozygotes portant une mutation d'un gène codant pour le récepteur de la prolactine, les auteurs révèlent chez ces souris un moins grand effet de l'accouplement sur la prolifération des cellules progénitrices. On sait que les souris mutantes hétéro- et homozygotes pour ce gène, comme les souris normales traitées par un antagoniste de la prolactine, ont des déficits du comportement maternel.

L'ensemble des données expérimentales montre que la production de nouveaux interneurones olfactifs est un mécanisme d'adaptation maternelle mis en route très tôt durant la gestation, et dépendant de la prolactine. La future mère est donc en mesure d'améliorer ses performances olfactives, ce qui l'aidera en particulier à mieux identifier ses petits.

\section{Kiyatkin EA, Mitchum RD Jr. : Fluctuations in brain temperature during sexual interaction in male rats : an approach for evaluating neural activity underlying motivated behavior. Neuroscience, 2003, 119 : 1169-1183.}

Partant du principe que l'activité métabolique dégage de la chaleur, les auteurs suggèrent que la mesure de la température intracérébrale permet d'identifier les zones cérébales actives durant certaines tâches : la température d'une zone active sera supérieure à celle qu'elle avait au repos, et la température d'une zone active sera supérieure 
à celle d'une région voisine inactive. La température de plusieurs noyaux cérébraux ( $\mathrm{n}$. accumbens, aire préoptique médiane de l'hypothalamus, hippocampe) est donc enregistrée et comparée à celle mesurée dans le muscle temporal durant l'activité sexuelle chez des rats mâles. La présentation d'une femelle augmente la température du muscle et de tous les noyaux enregistrés du cerveau de 1,5 à 2 ${ }^{\circ} \mathrm{C}$. Cette augmentation est plus rapide dans le cerveau que dans le muscle, s'accroît durant l'accouplement et culmine avec l'éjaculation. Après cette dernière, la température chute rapidement pour retrouver le niveau qu'elle avait avant la copulation, et se maintient à ce niveau durant toute la période réfractaire (le mâle ne s'intéresse pas à la femelle). Une nouvelle augmentation de la température est mesurée à la reprise de l'activité sexuelle.

Les données suggèrent que le comportement sexuel du mâle déclenche une augmentation générale soutenue de l'activité des noyaux cérébraux. Cette excitation centrale est causée par des stimuli particuliers qui ont une signification sexuelle, elle augmente au cours de la copulation et culmine avec l'éjaculation.

Les auteurs concluent que les variations de la température cérébrale sont non seulement une mesure fiable et sensible de l'activité d'une structure centrale, mais aussi un facteur puissant de contrôle des fonctions cérébrales, et une composante importante des mécanismes cérébraux à la base des comportements.

Wessells H, Hruby VJ, Hackett J, Han G, BalseSrinivasan P, Vanderah TW. : Ac-Nle-c[Asp-HisDphe-Arg-Trp-Lys]-NH2 induces penile erection via brain and spinal melanocortin receptors. Neuroscience, 2003, 118 : 755-762.

Les peptides de la famille des mélanocortines (alpha-, beta- et gamma-melanocyte stimulating hormone (MSH) et la corticotropine (ACTH)) régulent plusieurs fonctions telles que la prise alimentaire, la libido, l'érection et les réponses immunitaires. Chez le rat, alpha-MSH et ACTH délivrées par voie intracérébroventriculaire déclenchent des érections. Les sous-types MC3 et MC4 des récepteurs des mélanocortines sont présents dans le système nerveux central. Les auteurs de cet article explorent différents sites d'action (système nerveux central, tissus périphériques) des mélanocortines qui peuvent expliquer leur effet proérectile. Ils utilisent pour cela un agoniste hautement spécifique des sous-types $\mathrm{MC} 3$ et $\mathrm{MC} 4$ de leurs récepteurs, le Ac-Nle-c[Asp-His-Dphe-Arg-Trp-Lys]-NH2, ou MT-II. MT-II déclenche des érections lorqu'il est délivré par voie intracérébroventriculaire $(0,3-1-3 \mu \mathrm{g})$, intrathécale $(0,3-1-3$ $\mu \mathrm{g})$ et intraveineuse $(0,3-1-3 \mathrm{mg} / \mathrm{kg})$, mais pas lorsqu'il est injecté par voie intracaverneuse (0,1-1-10-100 $\mu \mathrm{g})$. Chez le rat éveillé, les effets proérectiles les plus puissants sont mesurés en réponse à l'injection intrathécale de MT-II : dans cette condition expérimentale, 8 érections sont enregistrées durant 90 minutes d'observation, contre 5 par voie intracérébroventriculaire, et 3 par voie intraveineuse. Les effets proérectiles de MT-II sont abolis par l'injection préalable de Ac-Nle-c[Asp-His-Dnal(2')-Arg-Trp-Lys]-NH2, ou SHU-9119, un antagoniste spécifique des sous-types MC3 et MC4 des récepteurs des mélanocortines.

Les résultats confirment un effet proérectile de MT-II et son site d'action central, probablement hypothalamique. Ils révèlent un effet proérectile du produit lorsqu'il agit sur la moelle épinière lombosacrée. Cette dernière est la source de l'innervation parasympathique sacrée du pénis. MT-II rejoint le petit groupe de substances proérectiles endogènes (ocytocine) ou exogènes (apomorphine) qui agissent dans le système nerveux central (moelle épinière, tronc cérébral, hypothalamus) pour exciter les circuits nerveux de l'érection. 